\title{
Waveform Control of Relativistic Electron Dynamics in Laser-Plasma Acceleration
}

\author{
Julius Huijts $\odot,{ }^{*}$ Lucas Rovige $\odot,{ }^{*}$ Igor A. Andriyash $\odot$, Aline Vernier, Marie Ouillé, Jaismeen Kaur $\odot$, \\ Zhao Cheng $\odot$, Rodrigo Lopez-Martens, and Jérôme Faure $\oplus^{\dagger}$ \\ LOA, CNRS, Ecole Polytechnique, ENSTA Paris, Institut Polytechnique de Paris, \\ 181 Chemin de la Hunière et des Joncherettes, 91120 Palaiseau, France
}

(Received 26 May 2021; revised 10 December 2021; accepted 11 January 2022; published 24 February 2022)

\begin{abstract}
The interaction of ultraintense laser pulses with an underdense plasma is used in laser-plasma acceleration to create compact sources of ultrashort pulses of relativistic electrons and $\mathrm{x}$ rays. The accelerating structure is a plasma wave, or wakefield, that is excited by the laser ponderomotive force, a force that is usually assumed to depend solely on the laser envelope and not on its exact waveform. Here, we use near-single-cycle laser pulses with a controlled carrier-envelope phase to show that the actual waveform of the laser field has a clear impact on the plasma response. The beam pointing of our relativistic electron beam oscillates in phase with the carrier-envelope phase of the laser, at an amplitude of $15 \mathrm{mrad}$, or $30 \%$ of the beam divergence. Numerical simulations explain this observation through asymmetries in the injection and acceleration of the electron beam, which are locked to the carrier-envelope phase. These results imply that we achieve waveform control of relativistic electron dynamics. Our results pave the way to high-precision, subcycle control of electron injection in plasma accelerators, enabling the production of attosecond relativistic electron bunches and $\mathrm{x}$ rays.
\end{abstract}

DOI: 10.1103/PhysRevX.12.011036

Subject Areas: Optics, Plasma Physics

\section{INTRODUCTION}

Waveform control has revolutionized several domains of laser-matter interaction, as it allows for an extremely high degree of control on electron dynamics. First applied in the frequency domain to drastically improve atomic clock precision [1], it was quickly adopted by the strong-field physics community to control electron dynamics in photoionization [2] and high-harmonic generation in gases [3,4] as well as laser-induced fragmentation of molecules [5]. Waveform control in laser-plasma interaction has been more difficult to achieve, because it requires few-cycle laser pulses with a stabilized carrier-envelope phase (CEP) [6] at intensities higher by several orders of magnitude. In laser-plasma interaction, CEP control was first demonstrated in overdense plasmas (i.e., solid targets) and at moderate intensity [7], with the final goal of producing isolated attosecond pulses of extreme ultraviolet radiation [8]. Recently, several experiments on solid targets at relativistic intensity have displayed CEP effects and their potential [9-12]. In experiments on

\footnotetext{
*These authors contributed equally to this work.

Corresponding author.

jerome.faure@ensta-paris.fr

Published by the American Physical Society under the terms of the Creative Commons Attribution 4.0 International license. Further distribution of this work must maintain attribution to the author(s) and the published article's title, journal citation, and DOI.
}

solid targets, the interaction takes place at the plasma surface where the CEP has a fixed value, but this is no longer true in underdense plasmas, as the laser can propagate in the medium. During propagation, the CEP slips, because the envelope travels at the group velocity $v_{g}$, which differs from the laser phase velocity $v_{\phi}$. The length scale over which dispersion changes the CEP by $2 \pi$ can then be estimated as $[13,14]$

$$
L_{2 \pi}=\frac{c}{v_{\phi}-v_{g}} \lambda_{0}
$$

where $c$ is the velocity of light and $\lambda_{0}$ is the laser wavelength. CEP-controlled electron dynamics can, thus, be observed only if the factor that governs these dynamics (e.g., electron beam injection) is localized to a fraction of $L_{2 \pi}$, such that an electron bunch can be associated to a specific CEP value. Additionally, a high level of control is required not only over the laser waveform, but also over the plasma parameters, as they strongly influence dispersion. Therefore, except for preliminary results [14] showing hints of CEP-dependent electron spectra in a laser-plasma accelerator (LPA), CEP effects in underdense laser-plasma interaction have remained elusive until now.

In a LPA, the laser-driven wakefield traps and accelerates electrons to relativistic energies over very short distances $[15,16]$. Wakefield excitation is particularly efficient when the laser pulse length $c \tau$ is resonant with the plasma wavelength, i.e., when $c \tau \simeq \lambda_{p} / 2$. Plasma electrons are then injected into and accelerated in the wakefield, forming high-quality, quasimonoenergetic electron beams [17-19]. 
While most LPAs are driven by laser pulses containing many optical cycles, few-cycle pulses can now be used to excite wakefields and accelerate electrons [20-23]. In this case, the usual framework of a cycle-averaged ponderomotive force [24] is not sufficient to describe the interaction. Instead, the actual waveform of the laser pulse needs to be considered, in particular, the phase between the envelope and the carrier wave (the CEP).

Theory and simulation studies show that the precise control of the laser waveform through the CEP can have a strong impact in LPAs. Simulations indicate that singlecycle laser pulses cause significant asymmetries of the plasma wakefield $[13,25]$. Nerush and Kostyukov [13] find that these asymmetries are due to the second-order term of the plasma response and are CEP dependent. As the laser pulse propagates in the plasma, the CEP slippage causes the wake to oscillate transversely on the length scale of $L_{2 \pi}$. As seen in recent simulations [26,27], the asymmetric plasma wakefield can, in turn, cause the off-axis injection of subfemtosecond electron bunches, with a collective, nonzero transverse momentum. As the electrons are accelerated, they oscillate collectively until they leave the plasma with an off-axis beam pointing that is CEP dependent. It is important to note that this effect is different from previously observed off-axis injection caused by laser pulse-front tilt [28], which can be satisfactorily explained within the framework of the cycle-averaged ponderomotive approximation.

In this paper, we observe this CEP-dependent beam pointing and give a detailed explanation through simulations that clearly show the CEP-dependent, off-axis injection of the electron beam. We describe the experimental setup and the obtained results in Sec. II and the simulations and their analysis in Sec. III.

\section{EXPERIMENT}

Our LPA is driven by laser pulses as short as 1.3 cycles, or 3.5 fs (FWHM in intensity) [14,21,22], generated by a double-chirped-pulse amplification system that makes use of spectral broadening through self-phase modulation in a helium-filled hollow-core fiber and subsequent

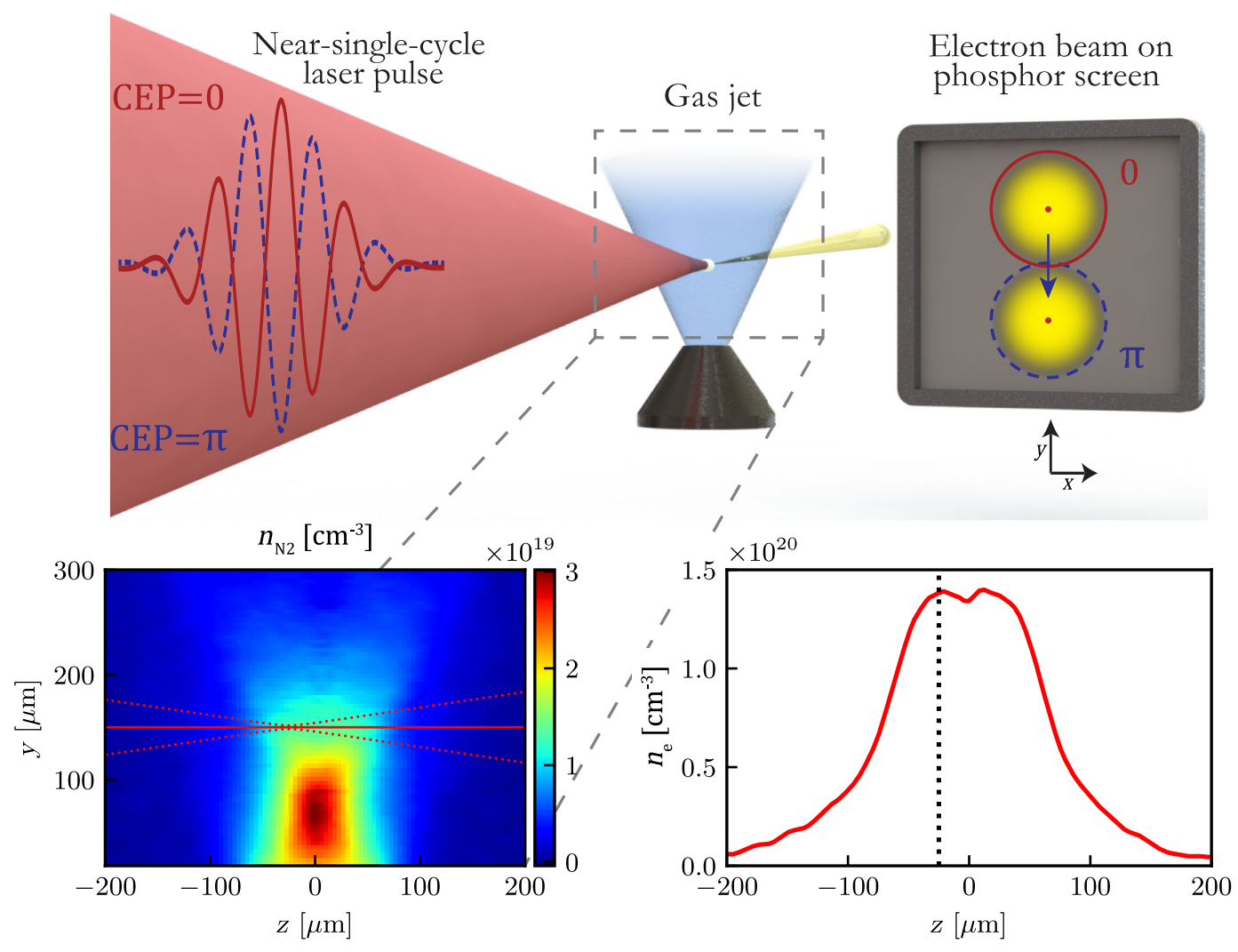

FIG. 1. Principle of the experiment. Upper: An intense, near-single-cycle laser pulse is focused into a gas jet, where it ionizes the gas and drives a plasma wake. Through laser wakefield acceleration, electrons are accelerated to relativistic energies. A phosphor screen is used to image the electron beam. The shape of the electric field of the laser pulse is controlled through the CEP. Varying the CEP in the experiment changes the pointing of the electron beam. Lower: 2D map of the gas density, retrieved from the phase map of an interferometry measurement (left). The height of the laser beam is indicated in red (150 $\mu \mathrm{m}$ from the jet exit). A lineout at this position of the plasma density (assuming full ionization of nitrogen up to $\mathrm{N}^{+5}$ ) is shown on the right. The dotted black line indicates the laser focal position. 
compression with chirped mirrors, as described elsewhere $[29,30]$. These pulses, with a final energy of $2.5 \mathrm{~mJ}$, are focused by an $f / 2$ off-axis parabola to a $2.7 \times 2.8 \mu \mathrm{m}$ focal spot with a vacuum peak intensity of $I=5 \times 10^{18} \mathrm{~W}$. $\mathrm{cm}^{-2} 25 \mu \mathrm{m}$ upstream of the center of a supersonic nitrogen gas jet (a "de Laval" nozzle [31] with a $60-\mu \mathrm{m}$ throat and $180-\mu \mathrm{m}$ exit diameter), as indicated in Fig. 1. The gas jet flows continuously while a pumping system keeps the residual gas pressure inside the chamber below $10^{-2}$ mbar. To obtain the plasma density profile, we illuminate the gas jet with a white light source and image it on a commercial quadriwave lateral shearing interferometer (SID4-HR, Phasics $[32,33])$. The interferometer provides a $2 \mathrm{D}$ phase map of the gas jet, and the $\mathrm{N}_{2}$ molecular density map is then retrieved via Abel inversion assuming cylindrical symmetry along the jet axis. The plasma density is then deduced assuming full ionization of nitrogen up to $\mathrm{N}^{5+}$, a molecule of $\mathrm{N}_{2}$ thus yielding ten electrons. The obtained peak plasma density of $n_{e}=1.4 \times 10^{20} \mathrm{~cm}^{-3}$ is achieved with a backing pressure of 15 bar. To distinguish CEP effects, it is important to keep the density profile as constant as possible throughout the experiment. A pressure controller ensures a subpercent stability on the backing pressure applied to the gas jet. In the gas jet, the laser pulse drives a plasma wake, in which electrons are accelerated to a few $\mathrm{MeV}$. The electron beam charge and distribution are measured with a calibrated $\mathrm{CsI}(\mathrm{Tl})$ phosphor screen imaged on a CCD camera. The position of the electron beam is determined as the point of maximum charge density after smoothing the image with a Gaussian kernel $(\sigma=2.8 \mathrm{mrad})$. The energy of the electrons is measured by inserting a removable spectrometer consisting of a 500- $\mu \mathrm{m}$ pinhole and two permanent circular magnets providing a 58-mT magnetic field. The minimum electron energy that can thus be measured is $0.5 \mathrm{MeV}$. During the experiments, the continuously flowing gas jet allows us to operate the laserplasma accelerator at the actual repetition rate of $1 \mathrm{kHz}$.

We are able to study the effect of the CEP on the electron beam as the CEP of the laser is stabilized using two feedback loops, to a shot-to-shot fluctuation between 240 and $550 \mathrm{mrad}$ rms depending on the target value (see the Appendix A).

As we vary the CEP, the pointing of the electron beam varies accordingly, as seen in Figs. 2(a) and 2(b) (and Supplemental Video 1 [34]). We define beam pointing as the deviation of the position of the electron beam from its average position during a CEP scan. The effect is significant: The amplitude of the oscillation is about $15 \mathrm{mrad}$, for a beam divergence of around $50 \mathrm{mrad}$, i.e., an approximately $30 \%$ change. The pointing varies in the plane of the laser polarization $(y)$, while in the perpendicular plane $(x)$ the beam pointing is constant except for a slow drift. Each acquisition is taken over $200 \mathrm{~ms}$ (i.e., 200 shots), which limits pointing jitter to below $2 \mathrm{mrad}$ rms. We can derive from Fig. 2(a) the maximum sensibility of the beam pointing to the CEP: $d \theta_{y} / d \phi=20 \mathrm{mrad} / \mathrm{rad}$. Therefore, in our (a)

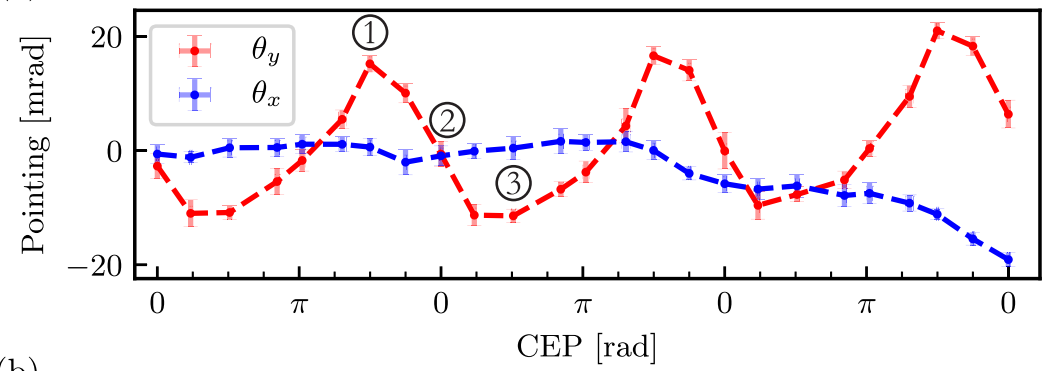

(b)
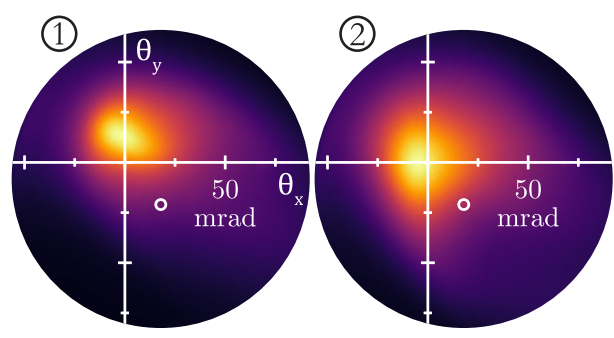

(c)

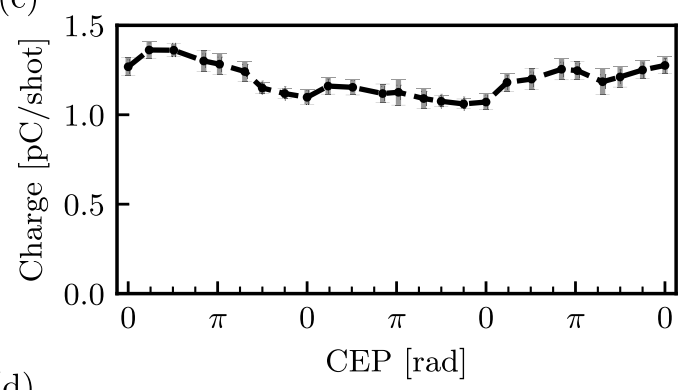

(d)

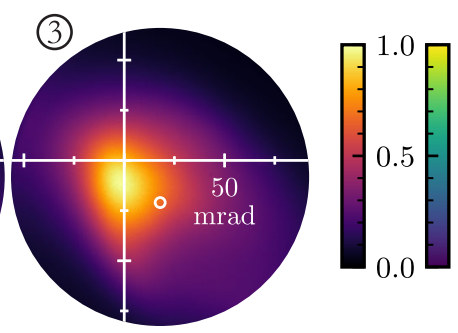

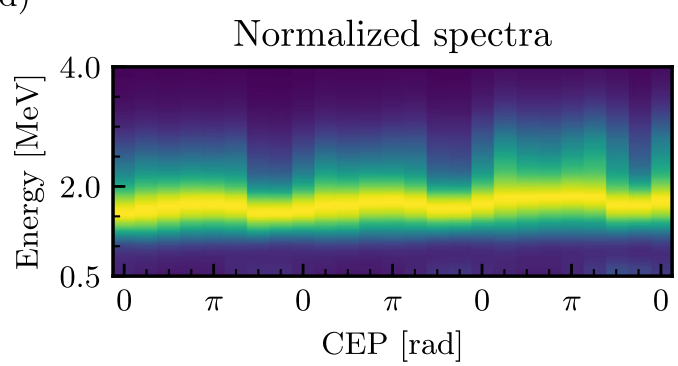

FIG. 2. Experimental results showing changes in electron beam parameters as the CEP is varied over three cycles of $2 \pi$. (a) The pointing of the electron beam in the plane of polarization $(y$, red) and in the perpendicular plane ( $x$, blue). (b) Typical images of the electron beam (acquired in $200 \mathrm{~ms}$, which corresponds to 200 shots) at a high (1), central (2), and low (3) beam pointing. The white circle indicates the part of the beam sampled by the pinhole of the electron spectrometer. (c) Evolution of the electron beam charge as a function of the CEP. (d) The normalized energy spectra of the electron beam as a function of the CEP. Each data point in (a) and (c) is the average of 20 acquisitions. The vertical error bars indicate the rms error of these acquisitions, yielding a sub-2-mrad pointing jitter (rms). The horizontal error bars indicate the rms error of the CEP stability, averaged over 200 shots (on the order of $40 \mathrm{mrad}$ ). 
case, without taking into account other sources of pointing jitter, it would be necessary to stabilize the CEP to $50 \mathrm{mrad}$ $\mathrm{rms}$ in order to obtain a stable beam with pointing variations of less than $1 \mathrm{mrad}$. The beam charge [Fig. 2(c)] is in the picocoulomb range and seems to show a small oscillation with the CEP of $8 \%$ peak to peak. This oscillation is not as evident as in the beam pointing, as it is dominated by a slow variation that is twice as large. The electron energy spectrum oscillates in phase with the CEP, albeit with a moderate amplitude of $5 \%$ of its mean value of $1.9 \mathrm{MeV}$; see Fig. 2(d). This spectral oscillation is due to a combination of two different effects: first, a direct effect of the CEP-i.e., a different CEP yields a different energy distribution-and, second, an indirect effect-as the beam pointing changes with the CEP, the electron beam position at the entrance of the spectrometer is different and a different part of the beam is sampled, leading to variations in the measured spectrum. This interpretation is supported by the analysis in Appendix B. Finally, CEP-dependent electron beam pointing is observed over a range of plasma densities (see Appendix C).

\section{SIMULATIONS AND DISCUSSION}

Particle-in-cell simulations using the spectral, quasicylindrical particle-in-cell code FBPIC [35] (see Appendix D) are carried out in order to reproduce the experimental results and gain more insight into the effect of the CEP on the injection and acceleration process. Typical simulated electron beams have a charge around $2.7 \mathrm{pC}$ and originate almost exclusively from self-injection (95\%), with a mean energy of $4.3 \mathrm{MeV}$. The difference between the experimental and simulated beam parameters can be explained by the fact that the simulation is run with an idealized laser pulse with Gaussian temporal and spatial profiles in order to focus on clarifying the underlying physical process. Figure 3(a) shows a first injection event that occurs off axis in the asymmetric wakefield, in the laser polarization plane. As the CEP slips by $\pi$, a second injection event occurs on the other side of the wakefield [Fig. 3(b)]. These two electron bunches are injected with opposite initial transverse momenta, and they end up with an opposite pointing when they exit the plasma [Fig. 3(c)].

For a more quantitative analysis, we define the wake asymmetry using the electron density transverse centroid, normalized to the laser waist $w_{0}: \Gamma_{y}=\left[\left(\int n_{e} y d y\right) /\right.$ $\left.\left(w_{0} \int n_{e} d y\right)\right]$. In Fig. 3(d), we first confirm that the wake oscillates transversely following the slippage of the CEP (red line), with a period corresponding to $L_{2 \pi}$. As the laser propagates, its interaction with the wakefield causes a

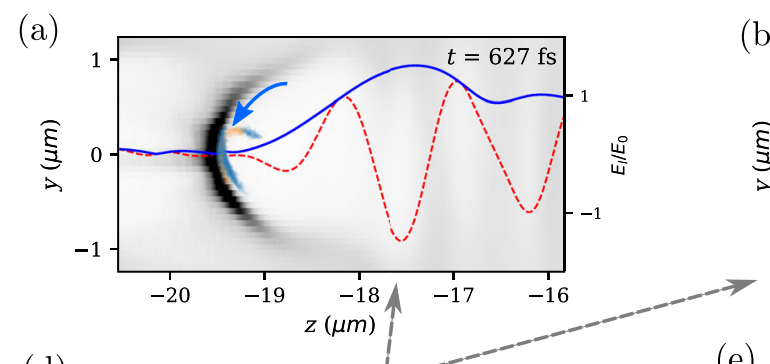

(d)

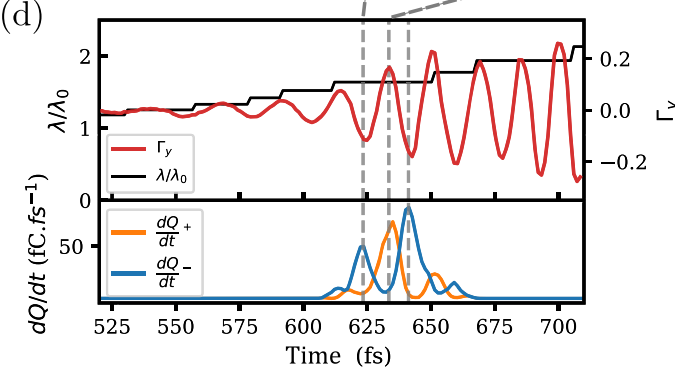

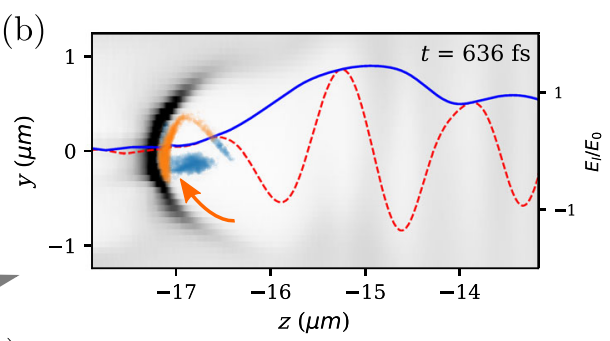

(e)

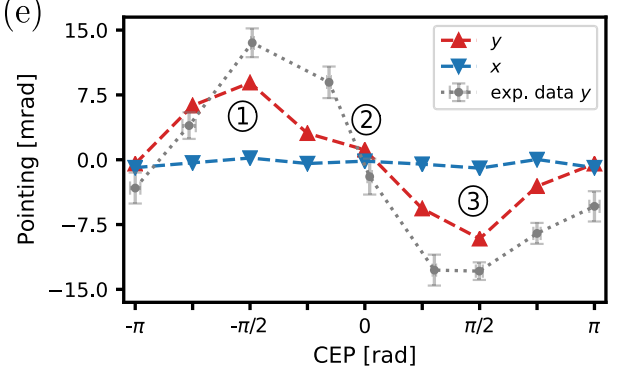

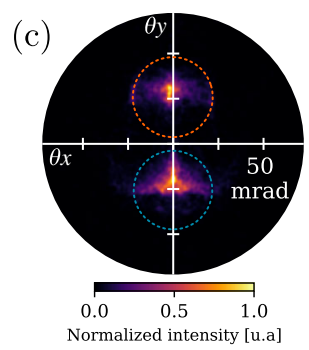

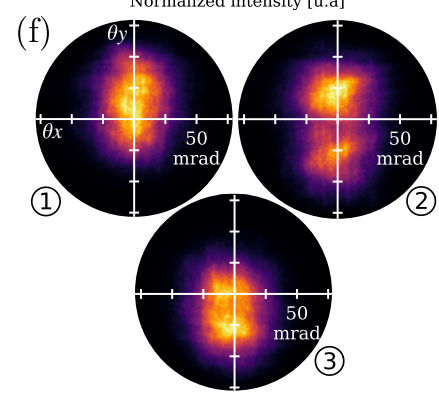

FIG. 3. Results of PIC simulations. (a),(b) Snapshots of the wakefield, for an initial CEP of $\pi$, at two different times, showing the injection of two separate bunches. Electron density in the $z-y$ plane is shown in gray, and injected electrons are displayed in orange (blue) when their pointing is positive (negative) at the end of the simulation. The normalized laser electric field $E_{l} / E_{0}=E_{l} /\left(m_{e} c \omega_{0} / e\right)(\mathrm{red}$ dashed line) and its envelope (blue solid line) are also shown. The arrows show the typical trajectories prior to injection for each case. (c) Simulated electron beam for an initial CEP of $\pi$ (single shot). (d) Wakefield oscillation in the polarization plane (red), peak wavelength of the laser normalized by the initial wavelength (black), and charge injection rate for the two electron populations shown in (a),(b) with corresponding colors, as a function of the simulation time for an initial CEP of $\pi$. The gray dashed lines highlight the three main injection events. (e) Electron beam pointing in the simulations in the directions parallel (red) and transverse (blue) to the laser polarization as a function of the initial CEP. The corresponding experimental data are shown in gray (the absolute value of the experimental CEP cannot be determined and is assumed so as to match the phase of the oscillations of the pointing in the simulations). (f) Simulated electron beams for initial CEP values of $-(\pi / 2), 0$, and $+(\pi / 2)$, which produce a high, centered, and low beam, respectively. The experimental beam pointing jitter is simulated by averaging 200 simulated shots with randomly generated beampointing variations following a normal distribution with a 15-mrad standard deviation (shot to shot) in both $x$ and $y$. 
strong redshift [36] (black line). This redshift shortens the oscillation period (as $L_{2 \pi} \propto \lambda^{-1}$ ) and enhances the wakefield asymmetry in the direction of polarization, following the scaling $\Gamma_{y} \propto E_{l 0}^{3} \lambda^{3}$ [13], where $E_{l 0}$ is the electric field peak amplitude. The redshift also lowers the laser group velocity, slowing down the wake, which lowers the threshold for electron injection $[14,37]$. The simulation shows that, when $\Gamma_{y}$ becomes large enough, the CEP-driven oscillatory motion of the plasma bubble allows trapping of electrons on the side the bubble has shifted to. As such, subfemtosecond electron bunches are injected at extrema of the bubble oscillation. This behavior is in agreement with the theory of electron injection in a transversely oscillating plasma bubble developed in Ref. [38]. The oscillation period at the time of injection is $L_{2 \pi}=6 \mu \mathrm{m}$ or $20 \mathrm{fs}$ (it is $L_{2 \pi}=10 \mu \mathrm{m}$ or $30 \mathrm{fs}$ before redshifting occurs). Note that the injection is very localized and consists in the injection of four sub-bunches, two of which contain most of the charge. These sub-bunches are as short as 0.9 fs (just after injection) and have a normalized emittance of $50 \mathrm{~nm} \cdot \mathrm{rad}$. This process clearly shows subcycle, waveform control over the electron dynamics: Electron injection is controlled by the wakefield asymmetry, which is itself controlled by the CEP.

The simulated beam in Fig. 3(c) corresponds to a single shot, while in the experiment the data are accumulated over 200 shots and average over a shot-to-shot pointing jitter that we estimate at $15 \mathrm{mrad} \mathrm{rms}$. To account for these fluctuations, we numerically add this jitter to the simulated data so that the two sub-bunches merge into a single larger divergence beam, so as to emulate the experimental behavior; see Fig. 3(f). A second effect that may merge the two sub-bunches in a single beam, not taken into account here, is that of space charge during propagation of the electron beam from the source to the detector. Simulations with the General Particle Tracer code [39] show that for a micrometric, 1-pC bunch at $2 \mathrm{MeV}$, space charge increases the divergence to tens of mrad FWHM, depending on the exact initial conditions. The PIC simulations also reproduce the dependence of the beam pointing with the CEP: The simulated beam oscillates by $9 \mathrm{mrad}$, which is comparable to the $15 \mathrm{mrad}$ obtained in the experiment; see Figs. 3(e) and 3(f). The overall change of the beam pointing with the CEP can be understood in the following way: The CEP determines the initial condition of the injected beams, namely, the initial longitudinal position in the plasma and initial transverse momentum $z_{0}, p_{y 0}$. The dynamics of the electron beam are then completely deterministic and consist of a transverse oscillation in the wakefield followed by several transverse oscillations in the laser field (see Supplemental Video 2 [34]). The final beam pointing is, therefore, completely determined by the CEP-controlled initial conditions.

\section{CONCLUSION AND OUTLOOK}

These results present experimental evidence that the injection of electrons and the beam parameters are governed directly by the laser waveform, demonstrating the importance of controlling the absolute phase of the field. For instance, in our experiment, stabilizing the beam pointing below $1 \mathrm{mrad}$ requires stabilizing the CEP with a precision better than $50 \mathrm{mrad}$. Simulations show that each injection event is localized at an extremum of the wakefield transverse oscillations and occurs on a few-micrometer length only. While in the present experiment several electron bunches are injected, a future goal is to restrict injection to a single subfemtosecond bunch. Injection of a single bunch could further reduce the initial phase space volume of the beam. If combined with phase space volume conservation during acceleration, this can lead to ultralow emittance beams while opening a path toward attosecond electron bunches. In our experiment, the near-single-cycle pulse is used for both injection and acceleration, but future experiments could combine a multicycle driver with a nearsingle-cycle injector in order to precisely control injection and generate subfemtosecond bunches as proposed in Ref. [38]. Injection schemes based on ionization [40,41] could also help in achieving the injection of a single subfemtosecond bunch, as ionization depends even more directly on the amplitude of the electric field [42]. Therefore, a CEP-stabilized single-cycle pulse could also be useful as an injector for plasma wakefield accelerators driven by particle beams [43]. In this context, ionization injection with subcycle, waveform-controlled, few-millijoule single-cycle laser pulses could provide attosecond and ultralow emittance relativistic electron beams $[44,45]$ for $\mathrm{x}$-ray production.

\section{ACKNOWLEDGMENTS}

This work was funded by the Agence Nationale de la Recherche under Contract No. ANR-20-CE92-0043-01. Financial support from the European Research Council (ERC Starting Grant No. FEMTOELEC 306708, ERC Advanced Grant No. ExCoMet 694596) is gratefully acknowledged. Numerical simulations were performed using HPC resources from GENCI-TGCC (Grand Équipement National de Calcul Intensif) (Grant No. 2020-A0090510062) with the IRENE supercomputer. This project has also received funding from the European Union's Horizon 2020 Research and Innovation program under Grant Agreement No. 101004730.

\section{APPENDIX A: CEP CONTROL}

The CEP stabilization is done in two loops, the first of which is a fast feedback loop on the oscillator which modulates the power of the pump laser (managed by an XPS800 by Menlo Systems, Garching, Germany). A second feedback loop stabilizes the CEP after amplification, spectral broadening, and compression. It uses a wedge reflection of our probe beam (a fraction of the main beam split off by a holed mirror and used for plasma diagnostics) 


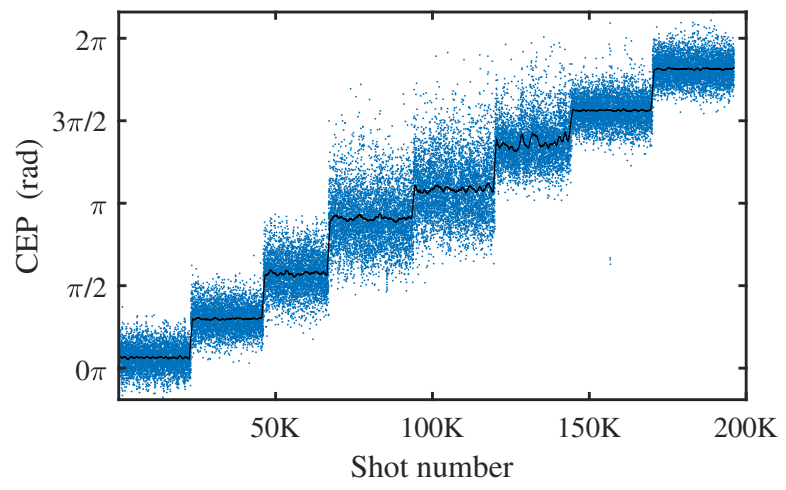

FIG. 4. Single-shot measurements (blue) and average over 200 shots (black) of the CEP during the first loop of the experimental CEP scan.

which is sent to an $f-2 f$ interferometer [46], which consists of a $\beta$-barium borate crystal for frequency doubling and a polarizer to project the fundamental and second-harmonic polarizations onto the same axis. The interference spectrum is analyzed shot to shot by a Fringeezz [47] (Fastlite, Antibes, France) to measure changes in the CEP. This measurement is fed back to an acousto-optic programmable dispersive filter (Fastlite, Antibes, France) in the first amplification stage to stabilize the CEP.

The CEP single-shot measurements for the first loop of the experimental scan as well as the values averaged on 200 shots are shown in Fig. 4. As our system allows one to measure only changes in CEP, and not the absolute $\mathrm{CEP}$, the reference value of $0 \mathrm{rad}$ at the start of a scan is arbitrary. When, during a scan, the CEP value is near $\pi$, the measurement becomes less accurate due to inherent limitations of the instrument (Fringeezz); therefore, the stability is significantly lower for CEP values near $\pi$. Indeed, the single-shot rms stability is around $240 \mathrm{mrad}$ when the CEP is stabilized near 0 and $550 \mathrm{mrad}$ when the CEP is stabilized near $\pi$. When averaging over 200 shots, as done for the electron beams, the rms stability is 30 mrad near a CEP value of 0 and $71 \mathrm{mrad}$ near $\pi$.

\section{APPENDIX B: ENERGY SPECTRA}

As the electron beam pointing varies with the CEP, the pinhole from the spectrometer does not sample the exact same point of the beam, as it is fixed [see Fig. 5(a) for a schematic drawing of the spectrometer]. This effect can be observed in the unnormalized spectra shown in Fig. 5(b), where the intensity of the measured spectrum varies significantly with the CEP, a variation much larger than the charge variations observed in Fig. 2(c). When the beam is off center with respect to the pinhole at the entrance of the spectrometer, less charge goes through and the spectrum intensity is, therefore, lower. This also means that the spectra are not measured at the exact same location for different CEP values, which could be responsible for (a)
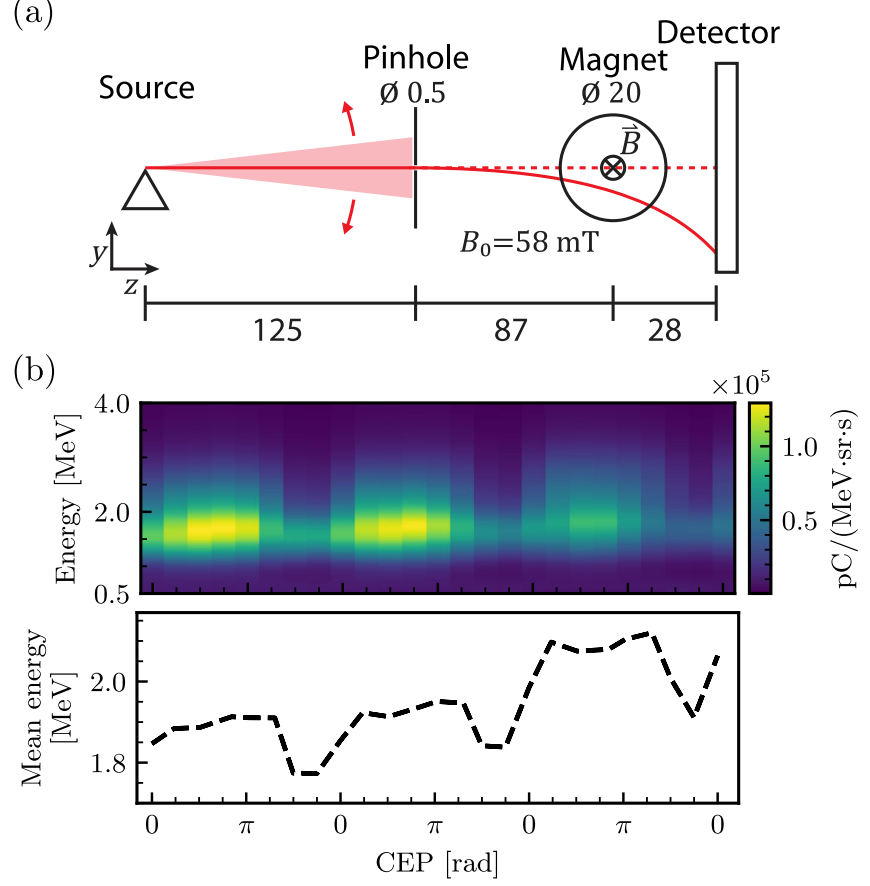

FIG. 5. (a) Schematic drawing of the spectrometer used in the experiment. The source, pinhole, magnet, and detector remain fixed throughout the CEP scan. The laser polarization and the CEP-dependent pointing oscillation (red arrows) are in the $y$ direction. Dimensions are in millimeters. (b, upper) The electron energy spectra from Fig. 2(d), without normalization. (b, lower) The mean electron energy as a function of the CEP.

differences in the electron energy. Experimentally, when cycling the CEP, the mean energy of the electron beam is found to vary by about $10 \%$.

We use our PIC simulations to quantify the effect of the oscillating beam position at the entrance of the spectrometer. We first look at the CEP dependence of the electron energy distribution; see Fig. 6(a). The CEP clearly affects the electron spectra, although the changes are relatively modest: The changes of the mean energy are only on the order of 5\% when considering the full electron beam; see the blue curve in Fig. 6(b). PIC simulations show that this can be explained by the CEP-induced changes of the injection longitudinal position. While it was already explained that the final pointing of a sub-bunch is determined by its initial transverse momentum $p_{y 0}$, simulations indicate that its final energy is determined by the initial longitudinal position $z_{0}$ which is also CEP dependent. Thus, sub-bunches are injected at CEP-dependent longitudinal positions, and, since the amplitude of the wake also slightly evolves with propagation, this leads to a slight dependence of the electron spectra to the initial laser CEP.

We model the experimental measurements by evaluating the energy distribution in a restricted 15-mrad aperture. This aperture is chosen larger than the one defined by the actual diameter of the entrance pinhole of the magnetic 


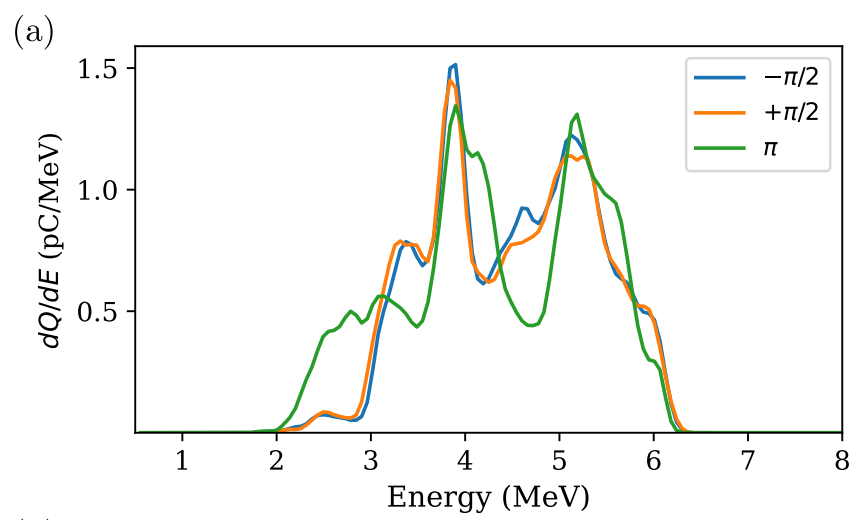

(b)

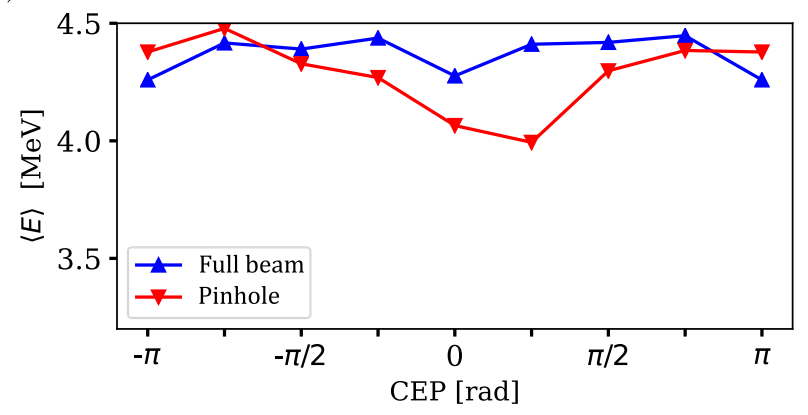

FIG. 6. (a) Simulated spectra for initial CEP values of $-(\pi / 2)$, $+(\pi / 2)$, and $\pi$. (b) Mean electron energy in the simulations as a function of the initial CEP, considering the full beam (blue) and the beam sampled through an off-center 15-mrad pinhole (red) in order to simulate the experimental configuration.

spectrometer, in order to mimic the electron beam-pointing fluctuation. It is also decentered vertically in order to match the relative position of the experimental one (see the white circle in Fig. 2 for the position of the pinhole relative to the beam). The red curve in Fig. 6(b) shows the variation of the mean energy when this effect is taken into account. In this case, the variation amounts to $11 \%$, which is close to the experimental variation. Moreover, while the periodicity of the variations of the simulated energy is $\pi$ for the full beam, it is $2 \pi$ when sampled through an off-center pinhole, because the upper half of the beam is never sampled, which corresponds to what is observed experimentally. Therefore, the observed experimental variations of the electron spectra can be attributed to a combination of an actual effect of the CEP on the energy of the electrons and a coupling between the energy and the point at which the beam is sampled.

\section{APPENDIX C: CEP EFFECTS AT DIFFERENT PLASMA DENSITIES}

We observe CEP effects in multiple scans at different plasma densities. For five scans, the electron beam is fully captured by our detector, such that pointing and divergence could be reliably determined. For these scans, we calculate the amplitude of the CEP-driven pointing oscillation $\delta \theta$, normalized to the beam divergence $\Delta \theta$ (Fig. 7). The ratio

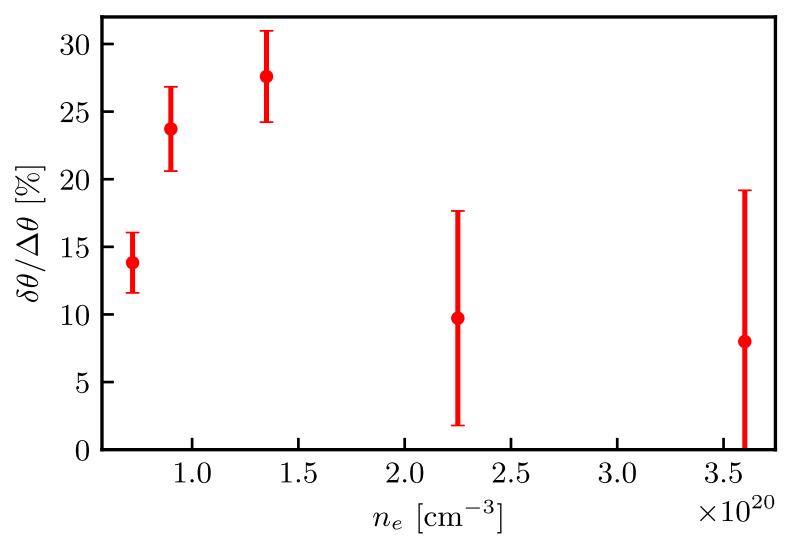

FIG. 7. The amplitude of the pointing oscillation $\delta \theta$, normalized to the beam divergence $\Delta \theta$, for different plasma densities. The error bars are calculated as the propagation of the statistical errors on $\delta \theta$ and $\Delta \theta$.

$\delta \theta / \Delta \theta$ represents the relative strength of the CEP effect for different plasma densities. The effect is more pronounced at moderate plasma densities, which can be explained from the dependence of $L_{2 \pi}$ (the length scale over which the CEP changes due to plasma dispersion) as the inverse of the plasma density. At low densities, as the CEP changes more slowly, electron injection can take place at a specific phase, and CEP effects are more pronounced. In addition, at higher plasma densities, the accelerator becomes less stable, which can be seen from the large error bars at $2.3 \times$ $10^{20}$ and $3.6 \times 10^{20} \mathrm{~cm}^{-3}$.

\section{APPENDIX D: SIMULATIONS}

For the simulations, we use a fully relativistic electromagnetic particle-in-cell code FBPIC [35] equipped with the pseudospectral analytical time domain (PSATD) quasicylindrical solver. The PSATD electromagnetic solver is free of numerical dispersion and provides a high-accuracy description of laser propagation and laser-particle interactions, while quasicylindrical geometry allows one to obtain a correct three-dimensional description at a moderate computational cost. The mesh used for simulations is $\Delta z=\lambda_{0} / 60$ and $\Delta r=5 \Delta z$. Five azimuthal Fourier modes are used to properly capture all asymmetries. The simulations are initialized with pure neutral nitrogen, and ionization is calculated with the Ammosov-DeloneKrainov model of tunnel ionization [48]. Atomic nitrogen is initialized using 96 macroparticles per $r-z$ cell, and each such macroparticle could produce up to seven macroparticles of electron species via ionization. Idealized Gaussian temporal and spatial laser profiles are used, with waist and pulse duration matching the experiment and a pulse energy of $2.3 \mathrm{~mJ}$. Dispersion in the plasma is precompensated by adding a $5-\mathrm{fs}^{2}$ positive chirp. For the simulated plasma profile, we use a combination of two super-Gaussian functions to fit the experimentally 
measured profile, with a peak density of $1.8 \times 10^{20} \mathrm{~cm}^{-3}$. The laser focus position is placed $25 \mu \mathrm{m}$ upstream of the center of the profile, like in the experiment.

[1] S. A. Diddams, T. Udem, J. Bergquist, E. Curtis, R. Drullinger, L. Hollberg, W. M. Itano, W. Lee, C. Oates, K. Vogel et al., An Optical Clock Based on a Single Trapped 199Hg + Ion, Science 293, 825 (2001).

[2] G. Paulus, F. Grasbon, H. Walther, P. Villoresi, M. Nisoli, S. Stagira, E. Priori, and S. De Silvestri, Absolute-Phase Phenomena in Photoionization with Few-Cycle Laser Pulses, Nature (London) 414, 182 (2001).

[3] A. Baltuška, T. Udem, M. Uiberacker, M. Hentschel, E. Goulielmakis, C. Gohle, R. Holzwarth, V. S. Yakovlev, A. Scrinzi, T. W. Hänsch, and F. Krausz, Attosecond Control of Electronic Processes by Intense Light Fields, Nature (London) 421, 611 (2003).

[4] M. Nisoli, G. Sansone, S. Stagira, S. De Silvestri, C. Vozzi, M. Pascolini, L. Poletto, P. Villoresi, and G. Tondello, Effects of Carrier-Envelope Phase Differences of FewOptical-Cycle Light Pulses in Single-Shot High-OrderHarmonic Spectra, Phys. Rev. Lett. 91, 213905 (2003).

[5] M. Kling, C. Siedschlag, A. J. Verhoef, J. Khan, M. Schultze, T. Uphues, Y. Ni, M. Uiberacker, M. Drescher, F. Krausz et al., Control of Electron Localization in Molecular Dissociation, Science 312, 246 (2006).

[6] D. J. Jones, S. A. Diddams, J. K. Ranka, A. Stentz, R. S. Windeler, J. L. Hall, and S. T. Cundiff, Carrier-Envelope Phase Control of Femtosecond Mode-Locked Lasers and Direct Optical Frequency Synthesis, Science 288, 635 (2000).

[7] A. Borot, A. Malvache, X. Chen, A. Jullien, J.-P. Geindre, P. Audebert, G. Mourou, F. Quéré, and R. Lopez-Martens, Attosecond Control of Collective Electron Motion in Plasmas, Nat. Phys. 8, 416 (2012).

[8] J. A. Wheeler, A. Borot, S. Monchocé, H. Vincenti, A. Ricci, A. Malvache, R. Lopez-Martens, and F. Quéré, Attosecond Lighthouses from Plasma Mirrors, Nat. Photonics 6, 829 (2012).

[9] D. Kormin, A. Borot, G. Ma, W. Dallari, B. Bergues, M. Aladi, I. B. Földes, and L. Veisz, Spectral Interferometry with Waveform-Dependent Relativistic High-Order Harmonics from Plasma Surfaces, Nat. Commun. 9, 4992 (2018).

[10] D. Cardenas, T. Ostermayr, L. Di Lucchio, L. Hofmann, M. Kling, P. Gibbon, J. Shreiber, and L. Veisz, Sub-cycle Dynamics in Relativistic Nanoplasma Acceleration, Sci. Rep. 9, 7321 (2019).

[11] O. Jahn, V. E. Leshchenko, P. Tzallas, A. Kessel, M. Krüger, A. Münzer, S. A. Trushin, G. D. Tsakiris, S. Kahaly, D. Kormin et al., Towards Intense Isolated Attosecond Pulses from Relativistic Surface High Harmonics, Optica 6, 280 (2019).

[12] F. Böhle, M. Thévenet, M. Bocoum, A. Vernier, S. Haessler, and R. Lopez-Martens, Generation of XUV Spectral Continua from Relativistic Plasma Mirrors Driven in the NearSingle-Cycle Limit, J. Phys. Photonics 2, 034010 (2020).
[13] E. N. Nerush and I. Y. Kostyukov, Carrier-Envelope Phase Effects in Plasma-Based Electron Acceleration with FewCycle Laser Pulses, Phys. Rev. Lett. 103, 035001 (2009).

[14] J. Faure, D. Gustas, D. Guénot, A. Vernier, F. Böhle, M. Ouillé, S. Haessler, R. Lopez-Martens, and A. Lifschitz, A Review of Recent Progress on Laser-Plasma Acceleration at $\mathrm{kHz}$ Repetition Rate, Plasma Phys. Controlled Fusion 61, 014012 (2019).

[15] T. Tajima and J. M. Dawson, Laser Electron Accelerator, Phys. Rev. Lett. 43, 267 (1979).

[16] E. Esarey, C. B. Schroeder, and W. P. Leemans, Physics of Laser-Driven Plasma-Based Electron Accelerators, Rev. Mod. Phys. 81, 1229 (2009).

[17] C. G. R. Geddes, C. Toth, J. van Tilborg, E. Esarey, C. B. Schroeder, D. Bruhwiler, C. Nieter, J. Cary, and W. P. Leemans, High-Quality Electron Beams from a Laser Wakefield Accelerator Using Plasma-Channel Guiding, Nature (London) 431, 538 (2004).

[18] J. Faure, Y. Glinec, A. Pukhov, S. Kiselev, S. Gordienko, E. Lefebvre, J.-P. Rousseau, F. Burgy, and V. Malka, A LaserPlasma Accelerator Producing Monoenergetic Electron Beams, Nature (London) 431, 541 (2004).

[19] S. P. D. Mangles, C. D. Murphy, Z. Najmudin, A. G. R. Thomas, J. L. Collier, A. E. Dangor, E. J. Divall, P. S. Foster, J. G. Gallacher, C. J. Hooker, D. A. Jaroszynski, A. J. Langley, W. B. Mori, P. A. Norreys, F. S. Tsung, R. Viskup, B. R. Walton, and K. Krushelnick, Monoenergetic Beams of Relativistic Electrons from Intense Laser-Plasma Interactions, Nature (London) 431, 535 (2004).

[20] K. Schmid, L. Veisz, F. Tavella, S. Benavides, R. Tautz, D. Herrmann, A. Buck, B. Hidding, A. Marcinkevicius, U. Schramm, M. Geissler, J. Meyer-ter-Vehn, D. Habs, and F. Krausz, Few-Cycle Laser-Driven Electron Acceleration, Phys. Rev. Lett. 102, 124801 (2009).

[21] D. Guénot, D. Gustas, A. Vernier, B. Beaurepaire, F. Böhle, M. Bocoum, M. Lozano, A. Jullien, R. Lopez-Martens, A. Lifschitz, and J. Faure, Relativistic Electron Beams Driven by $\mathrm{kHz}$ Single-Cycle Light Pulses, Nat. Photonics 11, 293 (2017).

[22] L. Rovige, J. Huijts, I. A. Andriyash, A. Vernier, M. Ouillé, Z. Cheng, T. Asai, Y. Fukuda, V. Tomkus, V. Girdauskas, G. Raciukaitis, J. Dudutis, V. Stankevic, P. Gecys, R. LopezMartens, and J. Faure, Optimization and Stabilization of a Kilohertz Laser-Plasma Accelerator, Phys. Plasmas 28, 033105 (2021).

[23] F. Salehi, M. Le, L. Railing, M. Kolesik, and H. M. Milchberg, Laser-Accelerated, Low-Divergence 15-MeV Quasimonoenergetic Electron Bunches at $1 \mathrm{kHz}$, Phys. Rev. X 11, 021055 (2021).

[24] P. Mora and T. M. Antonsen, Jr., Kinetic Modeling of Intense, Short Laser Pulses Propagating in Tenuous Plasmas, Phys. Plasmas 4, 217 (1997).

[25] A. Zhidkov, T. Fujii, and K. Nemoto, Electron Self-Injection during Interaction of Tightly Focused Few-Cycle Laser Pulses with Underdense Plasma, Phys. Rev. E 78, 036406 (2008).

[26] J. Huijts, I. A. Andriyash, L. Rovige, A. Vernier, and J. Faure, Identifying Observable Carrier-Envelope Phase Effects in Laser Wakefield Acceleration with Near-SingleCycle Pulses, Phys. Plasmas 28, 043101 (2021). 
[27] S. Xu, J. Zhang, N. Tang, S. Wang, W. Lu, and Z. Li, Periodic Self-Injection of Electrons in a Few-Cycle Laser Driven Oscillating Plasma Wake, AIP Adv. 10, 095310 (2020).

[28] A. Popp, J. Vieira, J. Osterhoff, Z. Major, R. Hörlein, M. Fuchs, R. Weingartner, T. P. Rowlands-Rees, M. Marti, R. A. Fonseca, S. F. Martins, L. O. Silva, S. M. Hooker, F. Krausz, F. Grüner, and S. Karsch, All-Optical Steering of Laser-Wakefield-Accelerated Electron Beams, Phys. Rev. Lett. 105, 215001 (2010).

[29] F. Böhle, M. Kretschmar, A. Jullien, M. Kovacs, M. Miranda, R. Romero, H. Crespo, U. Morgner, P. Simon, R. Lopez-Martens, and T. Nagy, Compression of CEPStable Multi-mJ Laser Pulses down to $4 \mathrm{fs}$ in Long Hollow Fibers, Laser Phys. Lett. 11, 095401 (2014).

[30] M. Ouillé, A. Vernier, F. Böhle, M. Bocoum, A. Jullien, M. Lozano, J.-P. Rousseau, Z. Cheng, D. Gustas, A. Blumenstein, P. Simon, S. Haessler, J. Faure, T. Nagy, and R. Lopez-Martens, Relativistic-Intensity Near-SingleCycle Light Waveforms at $\mathrm{kHz}$ Repetition Rate, Light Sci. Appl. 9, 47 (2020).

[31] K. Schmid and L. Veisz, Supersonic Gas Jets for LaserPlasma Experiments, Rev. Sci. Instrum. 83, 053304 (2012).

[32] J. Primot and L. Sogno, Achromatic Three-Wave (or More) Lateral Shearing Interferometer, J. Opt. Soc. Am. A 12, 2679 (1995).

[33] J. Primot and N. Guérineau, Extended Hartmann Test Based on the Pseudoguiding Property of a Hartmann Mask Completed by a Phase Chessboard, Appl. Opt. 39, 5715 (2000).

[34] See Supplemental Material at http://link.aps.org/ supplemental/10.1103/PhysRevX.12.011036 for a video of the experimentally measured electron beam and a video of the PIC simulation.

[35] R. Lehe, M. Kirchen, I. A. Andriyash, B. B. Godfrey, and J.-L. Vay, A Spectral, Quasi-cylindrical and DispersionFree Particle-in-Cell Algorithm, Comput. Phys. Commun. 203, 66 (2016).

[36] F. S. Tsung, C. Ren, L. O. Silva, W. B. Mori, and T. Katsouleas, Generation of Ultra-intense Single-Cycle Laser Pulses by Using Photon Deceleration, Proc. Natl. Acad. Sci. U.S.A. 99, 29 (2002).

[37] B. Beaurepaire, A. Lifschitz, and J. Faure, Electron Acceleration in Sub-relativistic Wakefields Driven by Few-Cycle Laser Pulses, New J. Phys. 16, 023023 (2014).
[38] J. Kim, T. Wang, V. Khudik, and G. Shvets, Subfemtosecond Wakefield Injector and Accelerator Based on an Undulating Plasma Bubble Controlled by a Laser Phase, Phys. Rev. Lett. 127, 164801 (2021).

[39] M. De Loos and S. Van Der Geer, in Epac 96: Proceedings of the Fifth European Particle Accelerator Conference, Sitges (Barcelona) (Institute of Physics Publishing, Bristol, 1996), p. 1241.

[40] C. McGuffey, A. G. R. Thomas, W. Schumaker, T. Matsuoka, V. Chvykov, F. J. Dollar, G. Kalintchenko, V. Yanovsky, A. Maksimchuk, K. Krushelnick, V. Y. Bychenkov, I. V. Glazyrin, and A. V. Karpeev, Ionization Induced Trapping in a Laser Wakefield Accelerator, Phys. Rev. Lett. 104, 025004 (2010).

[41] A. Pak, K. A. Marsh, S. F. Martins, W. Lu, W. B. Mori, and C. Joshi, Injection and Trapping of Tunnel-Ionized Electrons into Laser-Produced Wakes, Phys. Rev. Lett. 104, 025003 (2010).

[42] A. F. Lifschitz and V. Malka, Optical Phase Effects in Electron Wakefield Acceleration Using Few-Cycle Laser Pulses, New J. Phys. 14, 053045 (2012).

[43] I. Blumenfeld, C. E. Clayton, F.-J. Decker, M. J. Hogan, C. Huang, R. Ischebeck, R. Iverson, C. Joshi, T. Katsouleas, N. Kirby, W. Lu, K. A. Marsh, W. B. Mori, P. Muggli, E. Oz, R. H. Siemann, D. Walz, and M. Zhou, Energy Doubling of $42 \mathrm{GeV}$ Electrons in a Metre-Scale Plasma Wakefield Accelerator, Nature (London) 445, 741 (2007).

[44] B. Hidding, G. Pretzler, J. B. Rosenzweig, T. Königstein, D. Schiller, and D. L. Bruhwiler, Ultracold Electron Bunch Generation via Plasma Photocathode Emission and Acceleration in a Beam-Driven Plasma Blowout, Phys. Rev. Lett. 108, 035001 (2012).

[45] A. Deng et al., Generation and Acceleration of Electron Bunches from a Plasma Photocathode, Nat. Phys. 15, 1156 (2019).

[46] M. Kakehata, H. Takada, Y. Kobayashi, K. Torizuka, Y. Fujihira, T. Homma, and H. Takahashi, Single-Shot Measurement of Carrier-Envelope Phase Changes by Spectral Interferometry, Opt. Lett. 26, 1436 (2001).

[47] F. Lücking, V. Crozatier, N. Forget, A. Assion, and F. Krausz, Approaching the Limits of Carrier-Envelope Phase Stability in a Millijoule-Class Amplifier, Opt. Lett. 39, 3884 (2014).

[48] M. Ammosov, N. Delone, and V. Krainov, Tunnel Ionization of Complex Atoms and of Atomic Ions in an Alternating Electromagnetic Field, Sov. Phys. JETP 64, 1191 (1986). 